\title{
An Unusual Case of Nasal Pleomorphic Adenoma
}

\section{BACKGROUND:}

- Pleomorphic adenomas comprise $70 \%$ of parotid gland tumours and $50 \%$ of submandibular gland tumours ${ }^{1,2}$

- Up to $8 \%$ originate from minor salivary glands in the upper aerodigestive tract ${ }^{1,3-6}$

- $90 \%$ of nasal pleomorphic adenomas arise from the septum. However, most minor salivary glands occur in the lateral nasal wall ${ }^{3}$

- We present an unusual case of pleomorphic adenoma arising from the lateral wall of the nasal cavity.

\section{CASE REPORT - Presentation:}

- Symptoms: A 61 year-old male described a 6-month history of progressive right sided nasal obstruction, hyposmia, dysgeusia and offensive nasal discharge.

- Examination: Non-tender, polypoidal mass arising from the lateral wall of the right nostril.

- CT: Polypoidal soft tissue appeared to originate from the right middle meatus (Figures 1 and 2) (maximal diameter $2.3 \mathrm{~cm}$ ). There was no evidence of bony erosion, only moderate thinning and expansion of the adjacent right nasal bone.

\section{CASE REPORT - Imaging:}

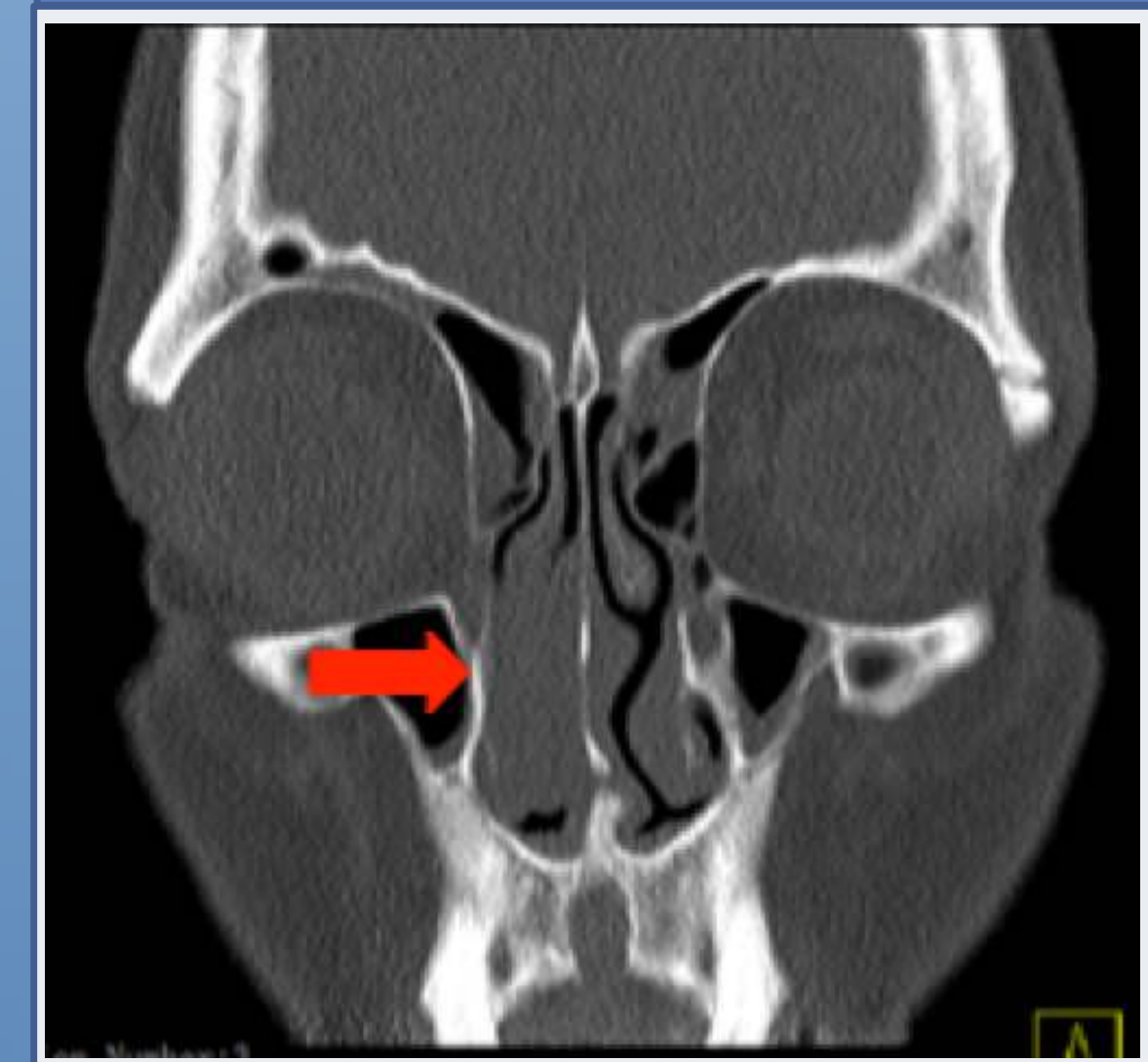

Figure 1 - Computed Tomography (CT) Scan: Coronal section demonstrating a right-sided polypoidal nasal mass (arrow)

Figure 2 - Computed Tomography (CT) Scan: Axial section demonstrating a rightsided polypoidal nasal mass (arrow)

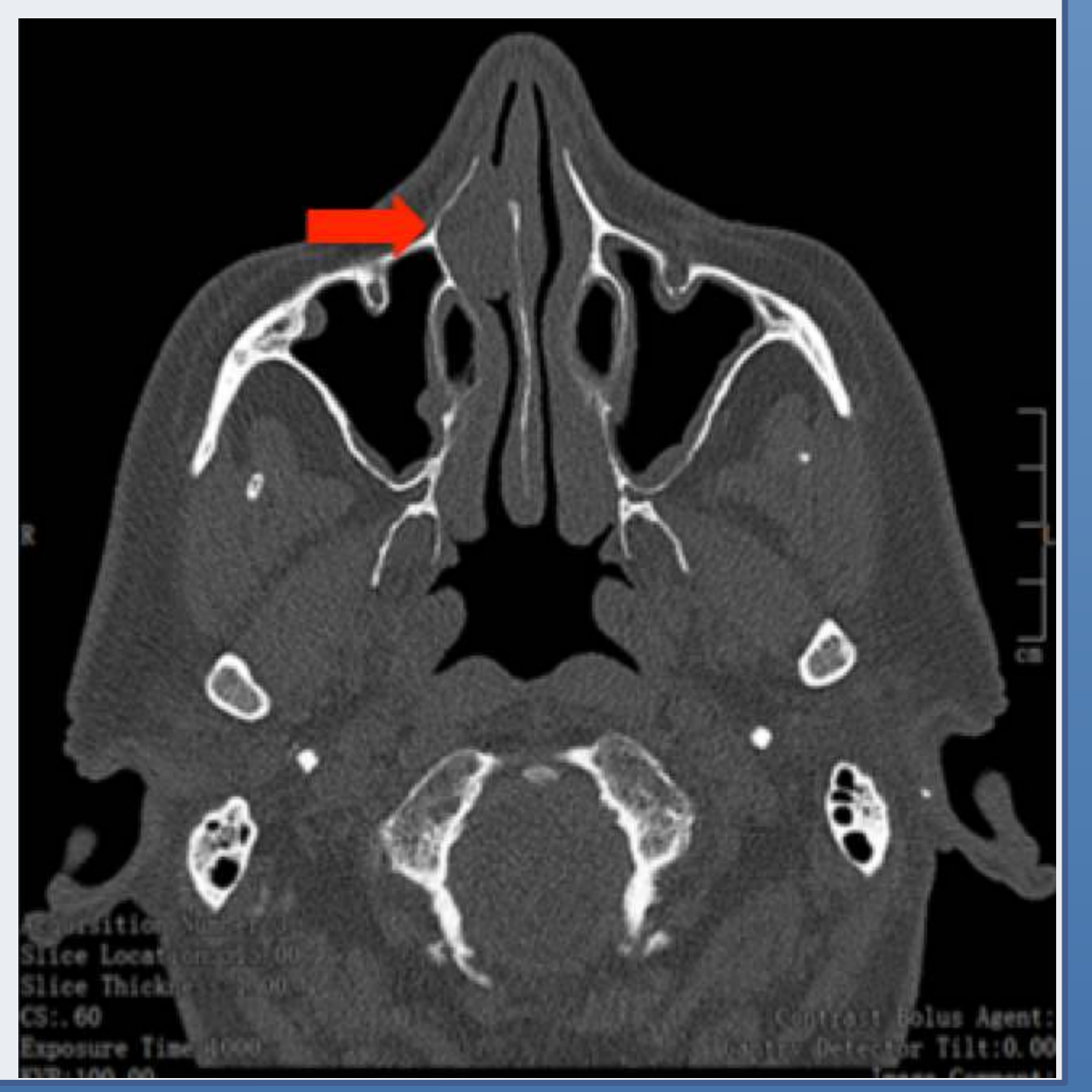

CASE REPORT - Management:

- FESS: A right nasal mass arose from the interior turbinate. An anterior ethmoidectomy was performed.

- Histology: Well-defined lesion composed of myxoid stroma, chondroid tissue, spindle and epithelial cells forming small groups of acini, glands and small sheets.

- Follow-Up: 6 months post-FESS, his hyposmia persisted, but he was otherwise asymptomatic, with no evidence of recurrence on flexible nasendoscopy.

\section{HISTOPATHOLOGY:}

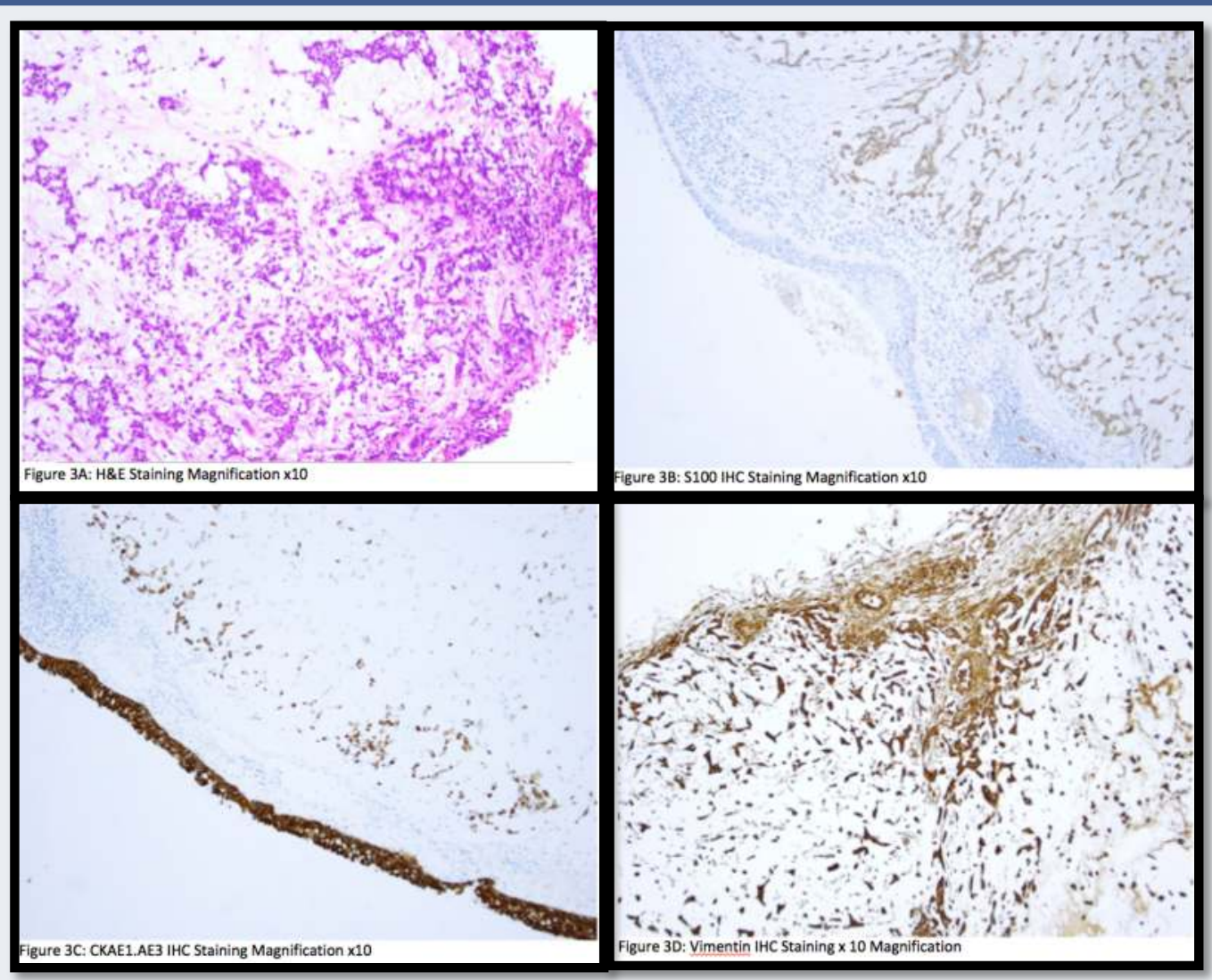

\section{DISCUSSION:}

- Although rare, pleomorphic adenomas can arise from the lateral nasal wall.

- Remnants of the vomeronsal organ, ectopic salivary gland or incomplete embryonic migration of the nasal buds may all account for their pathophysiology 3,7

- Typical symptoms include unilateral nasal obstruction, epistaxis, facial deformity and rhinorrhea $3,5,6,7$

- Epithelial tissue predominates nasal pleomorphic adenomas, with occasional stromal and myoepithelial elements. This makes differentiation from epithelial tumours difficult ${ }^{1,6}$

- Endoscopic wide local excision is the treatment of choice. Alternative approaches include lateral rhinotomy incisions ${ }^{1}$, external rhinoplasty ${ }^{1}$ or midfacial degloving 7

- Recurrence rates are 2.4-7.5\%7,8. We therefore recommend long-term follow up with regular examination and imaging.

\section{REFERENCES:}

